\title{
Cloning of the promoter region of a human gene, FOXL2, and its regulation by STAT3
}

\author{
YANGYANG HAN $^{1}$, TIANXIAO WANG ${ }^{1}$, SHUDONG $\mathrm{SUN}^{2}$, ZHAOHUI ZHAI ${ }^{1}$ and SHENGJIAN TANG ${ }^{1}$ \\ ${ }^{1}$ Plastic Surgery Institute, Weifang Medical University; \\ ${ }^{2}$ Department of Burns, Weifang People's Hospital, Weifang, Shandong 261053, P.R. China
}

Received July 23, 2016; Accepted May 5, 2017

DOI: $10.3892 / \mathrm{mmr} .2017 .6914$

\begin{abstract}
Forkhead box L2 (FOXL2) is a transcription factor, which is involved in blepharophimosis, ptosis, and epicanthus in versus syndrome (BPES), premature ovarian failure (POF), as well as almost all stages of ovarian development and function. FOXL2 has various target genes, which are implicated in numerous processes, including sex determination, cell cycle regulation and apoptosis and stress response regulation in mammals. However, studies regarding the upstream regulation of FOXL2 are limited. In the present study, the promoter of FOXL2 was successfully cloned and registered in Gen Bank, and a dual luciferase reporter (DLR) analysis demonstrated that the luciferase activity was significantly induced by the promoter of FOXL2. Subsequently, bioinformatics analysis indicated that FOXL2 may be regulated by STAT3, and this was confirmed by a DLR analysis and western blotting, using STAT3 inhibitors. Further study using real-time cellular analysis indicated that the viability of He La cells was markedly suppressed by STAT3 inhibitors. The present study demonstrated novel findings regarding the upstream regulation of FOXL2 expression and provide a new perspective for future studies in the field.
\end{abstract}

\section{Introduction}

Forkhead box L2 (FOXL2) is a gene encoding a fork head transcription factor, which belongs to the large family

Correspondence to: Dr Yangyang Han or Mr. Shengjian Tang, Plastic Surgery Institute, Weifang Medical University, 288 Weifang Shengli East Street, Weifang, Shandong 261053, P.R. China

E-mail: hanyy2009@163.com

E-mail: tangsjwfmc@163.com

Abbreviations: BPES, blepharophimosis, ptosis, and epicanthus inversus syndrome; DLR, dual luciferase reporter; FOXL2, forkhead box L2; GC, granulose cell; LUC, luciferase; RTCA, real-time cellular analysis; STAT3, signal transducer and activator of transcription 3

Key words: dual-luciferase reporter, forkhead box L2, promoter, signal transducer and activator of transcription 3, transcriptional regulation of forkhead/winged-helix transcription factors. It is a single-exon gene encoding a protein of 376 amino acids, and the protein contains a forkhead DNA-binding domain and a polyalanine tract of 14 residues (1). In humans, as well as mice and goats, the expression level of FOXL2 has been consistently observed in the developing eyelids, ovaries and the pituitary glands (2-4). Increasing evidence indicates that FOXL2 is an important transcription factor that is involved in various key life processes in humans, and its mutation may lead to diseases, such as blepharophimosis, ptosis, and epicanthus in versus syndrome (BPES) and premature ovarian failure.

FOXL2 is considered to be a possible inductor of female sex determination, actively repressing male genes, such as SRY-box 9 (SOX9) during development and more prominently later in adult life $(5,6)$. In addition, mutations of FOXL2 are responsible for BPES $(7,8)$, which includes eyelid and mild craniofacial defects that are either associated with primary ovarian insufficiency (type I) or not (type II) (9). Furthermore, a previous study demonstrated the involvement of FOXL2 in virtually all stages of ovarian development and function, as well as in granulose cell (GC)-associated pathologies (10). In the ovaries, FOXL2 is involved in the regulation of cholesterol and steroid metabolism, apoptosis, reactive oxygen species detoxification and cell proliferation (11). In a previous study in mice, FOXL2 was identified as the earliest ovarian maker, its expression being detectable from 12.0 d.p.c. (12).

However, the regulation of FOXL2 is less well understood and there are only a few studies regarding this. Dai et al (13) identified that microRNA-133b (miR-133b) bound to the 3'-untranslated region (3'-UTR) of FOXL2 mRNA, and miR-133b overexpression reduced the FOXL2 expression at the mRNA and protein levels. In addition, Luo et al (14) demonstrated that the 3'-UTR of mouse FOXL2 mRNA has one putative miR-133a binding site, and the expression level of FOXL2 was demonstrated to be downregulated by subsequent western blot analysis.

Signal transducer and activator of transcription 3 (STAT3) is a clinically significant latent transcription factor that is activated by multiple extracellular and intracellular signals, including the Janus kinase (JAK) family and the receptor tyrosine kinase (15). The phosphorylation of STAT3 results in its activation and nuclear translocation (16), and the activated STAT3 regulates gene expression in various biological 
processes, including proliferation, cell survival and inflammation (17). In addition, STAT3 is persistently activated in various human cancer cell lines, including human myeloma, breast and prostate cancer cells, and is involved in cancer development and progression, and contributes to cancer cell survival (18).

The present study predominantly focuses on the upstream transcriptional regulation of FOXL2. Following the successful cloning of the FOXL2 promoter region, it was analyzed using bioinformatics software and the results indicated that it may be regulated by STAT3. Subsequently, the the HeLa cells were exposed to the STAT3 inhibitors, and the dual luciferase reporter (DLR) analysis and western blot results demonstrated that FOXL2 was transcriptionally regulated by STAT3. Furthermore, using the xCELLigence real-time cellular analysis (RTCA) system, the growth and viability of the HeLa cells were demonstrated to be markedly suppressed compared with the control cells.

\section{Materials and methods}

Cell lines and treatments. The HeLa cell line, which was obtained from the Provincial Key Laboratory of Plastic and Microscopic Reconstructive Surgery Techniques of Shandong (Shandong, China), was cultured in high-glucose Dulbecco's modified Eagle's medium (DMEM; Hyclone; GE Healthcare Life Sciences, Logan, UT, USA) supplemented with $10 \%$ fetal bovine serum (Hyclone; GE Healthcare Life Sciences) at $37^{\circ} \mathrm{C}$ in a humidified environment with $5 \% \mathrm{CO}_{2}$.

STAT3 inhibitors. The STAT3 inhibitors, C188-9 and WP1066, were synthesized and supplied by Dr. Shaopeng Yuan (Institute of Materia Medica, Chinese Academy of Medical Sciences, Beijing, China), and dissolved in dimethyl sulfoxide (DMSO; Sigma-Aldrich; Merck KGaA, Darmstadt, Germany) as stock solution. This was serially diluted to the desired concentration using DMEM. The final concentration of DMSO in the culture systems was $<0.05 \%$.

Cloning and analysis of the promoter region of FOXL2. Based on the human mRNA sequence of FOXL2 [GenBank (https://www.ncbi.nlm.nih.gov/genbank/) accession no. NM023067], the human genome sequence was analyzed using the Basic Local Alignment Search Tool (BLAST) and the $\sim 2 \mathrm{~kb}$ region upstream of the predicted transcription start site was selected. Forward and reverse primers (pro-forward, 5'-CAGGGATTCTGCGGAAGTGC-3' and pro-reverse, 5'-GTTTCCCGAAGCACGACCC-3') were designed using Primer Premier software version 5.0 (Premier Biosoft International, Palo Alto, CA, USA) according to the sequences in NCBI (https://www.ncbi.nlm.nih.gov/). The promoter region of human FOXL2 $(\sim 1.9 \mathrm{~kb})$ was amplified by polymerase chain reaction (PCR) from human blood genomic DNA. PCR was carried out in a $25 \mu 1$ reaction containing $0.3 \mu 1$ EasyTaq DNA Polymerase (Beijing Transgen Biotech Co., Ltd., Beijing, China), with thermocycling conditions as follows: Initial denaturation at $94^{\circ} \mathrm{C}$ for $2 \mathrm{~min}$, followed by 30 cycles at $94^{\circ} \mathrm{C}$ for $30 \mathrm{sec}$, at $61^{\circ} \mathrm{C}$ for $30 \mathrm{sec}$ and at $72^{\circ} \mathrm{C}$ for $2 \mathrm{~min}$, with a final extension at $72^{\circ} \mathrm{C}$ for $20 \mathrm{~min}$. The PCR products were cloned into the pMD19-T simple vector (Takara
Bio, Inc., Otsu, Japan) and sequenced by Sangon Biotech Co., Ltd. (Shanghai, China) to confirm the resulting sequence. The cloned sequence was registered in GenBank (GenBank accession no. KR030055). The cis-regulatory elements in the promoter region of FOXL2 were predicted using TFSERACH (http://www.cbrc.jp/research/db/TFSEARCH.html) and Nsite (http://www.softberry.com/berry.phtml?topic $=$ nsite $\&$ group $=p$ rograms\&subgroup=promoter).

Plasmid construction. The promoter sequence of FOXL2 was isolated from the recombinant plasmid pMD19-T by PCR, as aforementioned, with the following primers synthesized using Primer Premier software version 5.0 (Premier Biosoft International): Pro-FE, 5'-ATGAGCTCCAGGGAT TCTGCGGAAGTGC-3' and Pro-RE, 5'-ATACTCGAG GTTTCCCGAAGCACGACCC-3', containing restriction enzyme cutting sites (underlined), and was confirmed by $10 \%$ agarose gel electrophoresis (Beijing Transgen Biotech Co., Ltd., Beijing, China) and sequencing by Sangon Biotech Co., Ltd. Following digestion with SacI and XhoI (Takara Bio, Inc.), the fragments were ligated into pGL3-Basic and pGL3-Enhancer vectors (Promega Corporation, Madison, WI, USA). Binary vectors containing ProFOXL2::luc were transformed into Escherichia coli DH5a (Beijing Transgen Biotech Co., Ltd.) using the freeze-thaw method. The recombinant plasmid was extracted using a Mini Plasmid kit (Tiangen Biotech Co., Ltd., Beijing, China) according to the manufacturer's protocol.

Transient transfection and luciferase reporter assays. For transient transfection using Invitrogen Lipofectamine ${ }^{\circledR} 3000$ (Thermo Fisher Scientific, Inc., Waltham, MA, USA), the Lipofectamine $^{\circledR} 3000$ Reagent was diluted in Opti-MEM medium (Thermo Fisher Scientific, Inc.), and the master plasmid was prepared by diluting the recombinant pGL3-ProFOXL2 plasmid in Opti-MEM medium and adding P3000 Reagent (Thermo Fisher Scientific, Inc.). Subsequently, diluted plasmid was added to each tube of diluted Lipofectamine ${ }^{\circledR} 3000$ Reagent (1:1 ratio) and incubated for $5 \mathrm{~min}$ at room temperature. Different plasmid-lipid complexes $(0.25 \mu \mathrm{g} /$ well $)$ were then added to HeLa cells plated in 12 -well plates $\left(70-90 \%\right.$ confluent) at $37^{\circ} \mathrm{C}$ separately. All cells were co-transfected with the Renilla luciferase reporter plasmid (5 ng/well, pRL-TK; Promega Corporation) as a control for transfection efficiency. Luciferase (LUC) activity was assayed $48 \mathrm{~h}$ after transfection using the Dual-Luciferase Reporter Assay system (Promega Corporation) and measured using a Lumat 3 LB9508 luminescence counter (Berthold Technologies GmbH \& Co. KG, Bad Wildbad, Germany). A minimum of three transfection assays was performed to obtain statistically significant data.

Western blot analysis. HeLa cells $\left(1.5 \times 10^{6}\right.$ cells/well $)$ in 6 -well plates, which had been grown under normal conditions, were treated with different concentrations $(1,2.5,5$ or $10 \mu \mathrm{M}) \mathrm{WP} 1066$ for $48 \mathrm{~h}$; cells treated with DMSO to a final concentration at $1 \%$ served as the control group. Proteins were collected using $150 \mu \mathrm{l} /$ well radioimmunoprecipitation assay lysis buffer (Thermo Fisher Scientific, Inc.), frozen for $30 \mathrm{~min}$ and stored at $-20^{\circ} \mathrm{C}$. 
For western blot analysis, proteins that had been extracted from HeLa cells were boiled in 1X SDS-PAGE loading buffer (Takara Bio, Inc.). Protein concentrations were measured using a bicinchoninic acid protein assay and equal amounts of extracted protein samples (30 $\mu 1$ lysate/lane) were separated on $10 \%$ SDS-PAGE, as previously described (19) and transferred onto polyvinylidene difluoride membrane (EMD Millipore, Billerica, MA, USA). The membrane was blocked using skim milk for $2.5 \mathrm{~h}$ at room temperature. Immunoblotting was performed using the following primary antibodies for $3 \mathrm{~h}$ at room temperature: Anti-FOXL2 (cat no. ab188584; 1:5,000; Abcam, Cambridge, MA, USA), anti-GAPDH (cat no. ab181602; 1:10,000; Abcam). Membranes were then incubated with horseradish peroxidase (HRP)-conjugated goat anti-rabbit secondary antibodies (cat no. BA1054; 1:3,000; Wuhan Boster Biological Technology, Ltd., Wuhan, China) for $2.5 \mathrm{~h}$ at room temperature. An enhanced HRP-diaminobenzidine chromogenic substrate kit (Tiangen Biotech Co., Ltd., Beijing, China) was used for immunodetection, according to the manufacturer's instructions. Blots were semi-quantified by densitometry using the FluroChem R system and AlphaView software (ProteinSimple; Bio-Techne, Minneapolis, MN, USA).

Real-time cell viability assay by RTCA. The cytotoxic effect of C188-8 or WP1066 was determined using the xCELLigence system (Roche Diagnostics, Basel, Switzerland), which measures cell status (provided as cell index) utilizing an electric current to determine cellular attachment to an electrode-containing plate. Cells $\left(5 \times 10^{3}\right.$ cells/well; $\left.100 \mu \mathrm{l}\right)$ were seeded in the electrode-containing plate (e-plate) and incubated for $24 \mathrm{~h}$ at $37^{\circ} \mathrm{C}$ in a humidified environment with $5 \% \mathrm{CO}_{2}$. The inhibitors of STAT3, C188-9 or WP1066, were added (at a concentration of 5 or $10 \mu \mathrm{M}$ ) and the electrical impedance, caused by cell adhesion to the plate (which is directly proportional to cellular viability) was numerically reported. Cells treated with DMSO served as a negative control. Data from the xCELLigence system was collected using the software provided with the system, and values were normalized at the point of treatment with compounds for all cell lines.

Statistical analysis. Statistical analysis was performed using the Data Processing System software version 7.0 developed by Zhejiang University (Hangzhou, China). Data are expressed as the mean \pm standard error of the mean of at least 3 independent experiments. The statistical significance of the differences between groups was evaluated using one-way analysis of variance followed by a post hoc Duncan's test for multiple comparisons. $\mathrm{P}<0.05$ was considered to indicate a statistically significant difference.

\section{Results}

Cloning and BLASTing of the 5' flanking region of FOXL2. In order to characterize the transcriptional regulation mechanism of FOXL2, the promoter region of FOXL2 was cloned. Using the genomic DNA of normal human blood samples (Fig. 1A) as templates, the 1,931-bp 5' region upstream of the transcription start site was cloned (Fig. 1B) and registered in Gen Bank
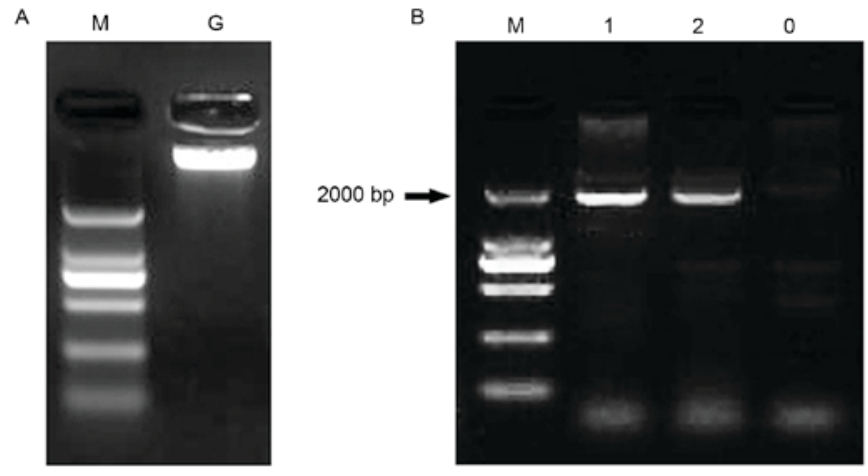

Figure 1. Cloning of FOXL2 promoter from healthy human blood. (A) Extraction of genomic DNA from normal human blood. Lane M, DL2000 DNA marker $(2,000,1,000,750,500,250$ and $100 \mathrm{bp})$; lane $\mathrm{G}$, normal genomic DNA. (B) Cloning of the promoter of FOXL2 using normal human genomic DNA. Lanes 1 and 2, the 1,931-bp upstream region of FOXL2; lane 0 , negative control; FOXL2, forkhead box L2.

(Gen Bank accession no. KR030055), and the BLASTing result indicated that the 5 ' flanking region of FOXL2 was successfully cloned (data not shown).

LUC activity was significantly induced by the cloned 5' flanking region. To assess whether the cloned 5' flanking region was actually the promoter of FOXL2, the region was fused to pGL3 (pGL3-Basic and pGL3-Enhancer) vectors, which contain luciferase reporter genes (Fig. 2A). The digested result of double restriction enzymes is presented in Fig. 2B, which demonstrates that the recombinant vectors containing the 1,931-bp 5' flanking region of FOXL2 were successfully constructed (Fig. 2B).

Then the constructed vectors were transient transfected into HeLa cells, and the LUC activity was measured using a DLR assay system. As shown in Fig. 3, the relative LUC activities in pGL3-Basic Pro and pGL3-Enhancer Pro were significantly higher than the negative control (empty vector of pGL3-Basic and pGL3-Enhancer). Thus, the results in Figs. 2 and 3 indicate that the 1,931-bp fragment was the promoter of FOXL2 and it significantly induces the expression of the luciferase gene.

Promoter of FOXL2 has STAT3 binding sites, and the expression level of FOXL2 was down regulated by STAT3 inhibitor. Using TFSERACH and Nsite, the Cis-regulatory elements in the promoter of FOXL2 were predicted, and the result demonstrated STAT3 binding sites (TGTCTTCCCAGTCTGT) in the promoter region (data not shown). To verify this prediction, two STAT3 inhibitors (C188-9 and WP1066) were used to treat the cells that were transfected with pGL3-Basic Pro and pGL3-Enhancer Pro vectors, and then the LUC activities were measured (Fig. 4A). As shown in Fig. 4A, the LUC activity with WP1066 treatment was significant lower than that without WP1066, while the cell treatment with C188-9 did not show any obvious changes.

Subsequently, further detection was performed using western blotting to verify the regulation of FOXL2 by STAT3. The expression level of FOXL2 protein decreased markedly following treatment with different concentrations of STAT3 inhibitor when compared with the control (Fig. 4B). Thus, the results confirmed that the promoter region of FOXL2 contains STAT3 binding 
A
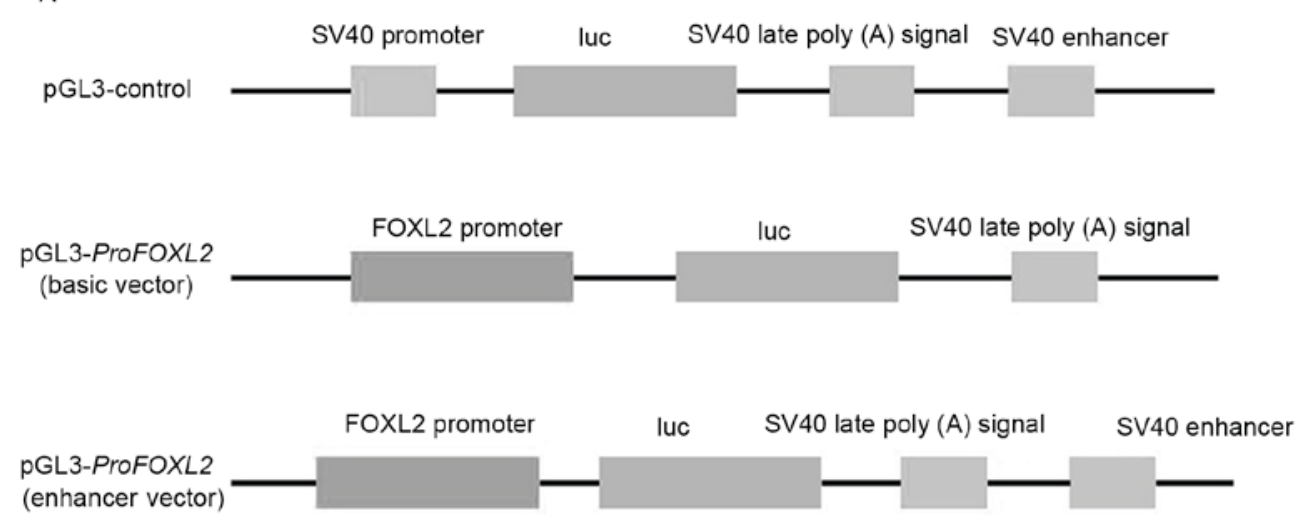

B
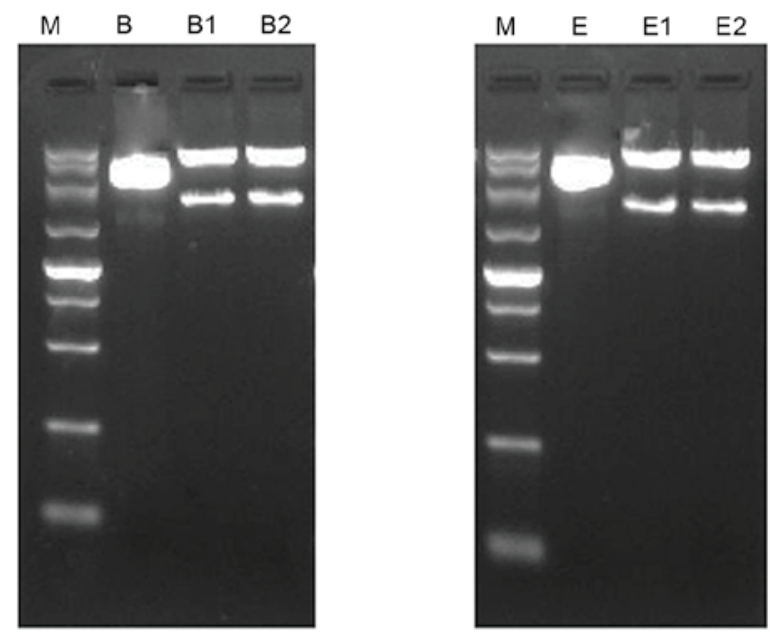

Figure 2. Construction of vectors containing the promoter of FOXL2 and the luciferase reporter gene. (A) Schematic representation of the pGL3-ProFOXL2 basic and enhancer fusion constructs, and the pGL3-Control construct. (B) Electrophoresis results of pGL3-ProFOXL2 basic vector (left) and pGL3-ProFOXL2 enhancer vector (right) following digestion by double restriction enzymes (SacI and XhoI); the basic and enhancer vectors without the fusion promoter served as the negative control. Lane M, DL5000 (5,000, 3,000, 2,000, 1,500, 1,000, 750, 500, 250 and 100 bp); lane B, pGL3 basic vector; lanes B1 and B2, pGL3-ProFOXL2 basic vector; lane E, pGL3 enhancer vector; lanes E1 and E2, pGL3-ProFOXL2 enhancer vector. FOXL2, forkhead box L2; luc, luciferase.

sites, and the expression level of FOXL2 was down-regulated by the inhibitor of STAT3 in protein level (Fig. 4).

Cell growth was repressed by the inhibitor of STAT3. For further analysis of the influence of the STAT3 inhibitor, HeLa cells, which were plated onto an e-plate, were treated with different concentration inhibitors of STAT3, and the real-time cell viabilities (cell index) were detected (Fig. 5). As predicted, cell indexes demonstrated a marked decline after the normal HeLa cells were exposed to the inhibitors, C188-9 and WP1066, compared with cells that were treated with the same volume of medium or DMSO. WP1066 markedly reduced cell growth (0.3-0.4 cell index) when compared with C188-9 (0.4-0.55 cell index) at $6 \mathrm{~h}$ after treatment (Fig. 5A and B). However, cells treated with DMSO also exhibited a decreased normalized cell index compared with control cells.

\section{Discussion}

As a transcription factor, FOXL2 is highly conserved among vertebrates, and has emerged as a key factor in ovarian biology, for example during early ovarian development and female sex determination, as well as in postnatal somatic cell differentiation and follicle maintenance (20). In addition, mutations of FOXL2 are responsible for the BPES (13), and previous studies have demonstrated that FOXL2 has various potential direct and indirect transcriptional targets, including SOX9 (21), cytochrome P450 family 19 subfamily A member 1 (CYP19A1) (22), SMAD family member 3 (SMAD3) (6), follistatin (FST) (21), steroidogenic acute regulatory protein $(S T A R)$ (23), follicle stimulating hormone beta subunit $(F S H B)(24)$, gonadotropin releasing hormone receptor $(G N R H R)(25)$, superoxide dismutase 2 (SOD2) and sirtuin 1 (SIRT1) (26). However, studies regarding the underlying mechanisms of FOXL2 regulation are limited.

In the present study, the promoter region of FOXL2 was cloned (Fig. 1) and the binary vector containing the luciferase gene was successfully constructed (Fig. 2). DLR analysis indicated that LUC activity was significantly induced when compared with the negative control vector (Fig. 3). The bioinformatics analysis then indicated that FOXL2 may be regulated by STAT3.

As a transcription factor, STAT3 is constitutively expressed in a wide variety of tissue types, and is activated by various 


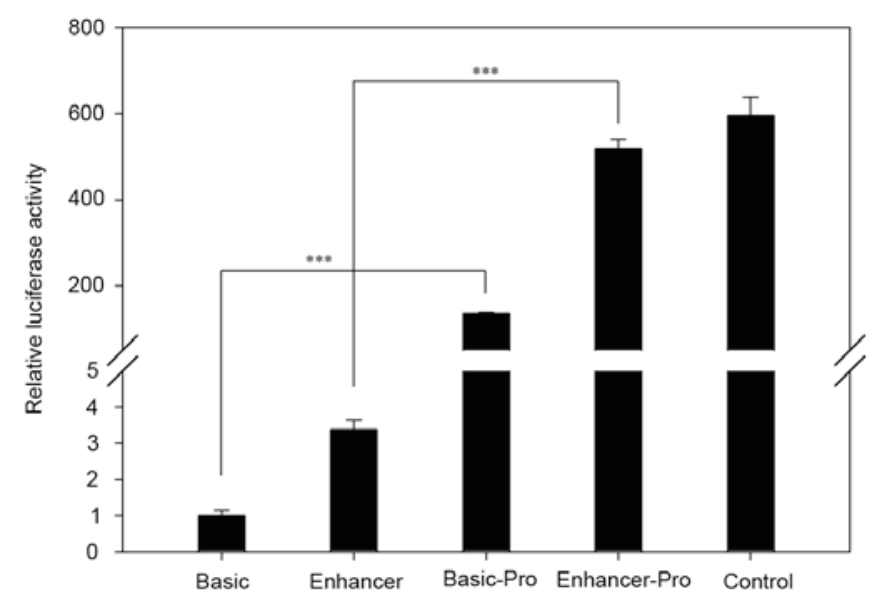

Figure 3. Luciferase activity was induced significantly by the promoter of FOXL2. HeLa cells were transfected with pGL3-Basic or pGL3-Enhancer vector plasmids containing the fusion promoter of FOXL2 (Basic-Pro and Enhancer-Pro), the pGL3-Basic or -Enhancer empty vector plasmids were also transfected as the negative control, and the pGL3-Control vector was transfected as the positive control. All HeLa cells were cotransfected with the Renilla luciferase reporter plasmid (pRL-TK) as a control for transfection efficiency using Lipofectamine ${ }^{\circledR} 3000$ reagent. After 48 h, luciferase assays were performed and normalized by constitutive Renilla luciferase. Each column represents the mean \pm standard error of the mean of at least three replicates and the entire experiment was repeated 3 times. ${ }^{* * * *} \mathrm{P}<0.001$, as indicated. FOXL2, forkhead box L2.

cytokines and growth factors, as well as proto-oncogenes and oncogenes $(27,28)$. The present results confirmed that the activity of luciferase, which fused to the promoter of FOXL2, is significantly suppressed by the STAT3 inhibitor WP1066 (Fig. 4A). This result implied that FOXL2 may be transcriptionally regulated by STAT3, which was further demonstrated by the western blot result (Fig. 4B). To the best of our knowledge, this is a novel finding on the upstream regulation of FOXL2. However, the binding sequence of STAT3 (data not shown) in the promoter region of FOXL2 was only predicted using the bioinformatics software, thus further evidence is required to confirm the results in Fig, 4, and further experiments have been conducted in our next paper.

Although the expression level of the FOXL2 promoter was suppressed significantly by WP1066, another inhibitor of STAT3, C188-9, did not significantly suppress LUC activity (Fig. 4A). As a transcription factor, STAT3 is activated by phosphorylation of tyrosine residue, after which it dimerizes and translocates into the nucleus. WP1066 is a novel inhibitor of STAT3 that is structurally associated with AG490, which is a kinase inhibitor and originally selected from a group of tyrphostins screened for their ability to block JAK-2 activity, but is significantly more potent and active $(29,30)$. While C188-9 (a second-generation compound of C188), which was identified to be active in inhibiting granulocyte colony stimulating factor, induced STAT3 phosphorylation (31). Thus, the current results indicate that FOXL2 was transcriptionally regulated by STAT3, via the JAK2-STAT3 signaling pathway.

DMSO is an important aprotic solvent that solubilizes a wide variety of otherwise poorly soluble polar and nonpolar molecules (32). In the present study, inhibitors of STAT3 (WP1066 and C188-9) were dissolved in DMSO as stock solution. The RTCA using the xCELLigence system indicated

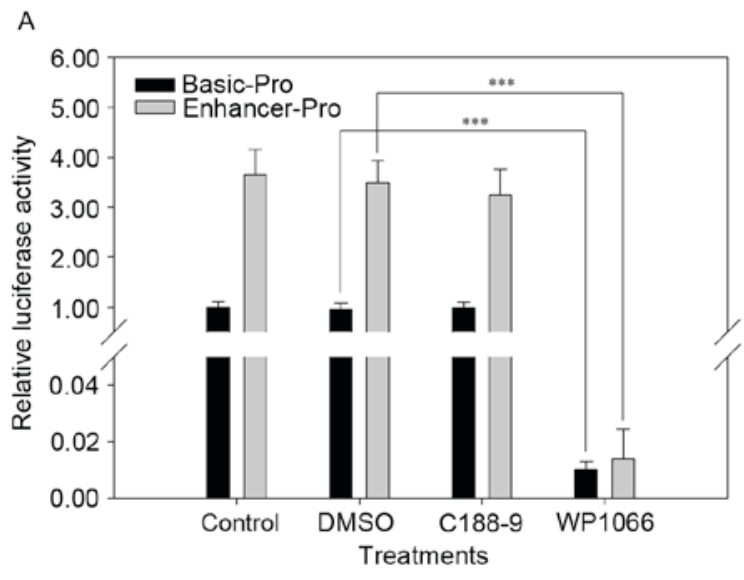

B
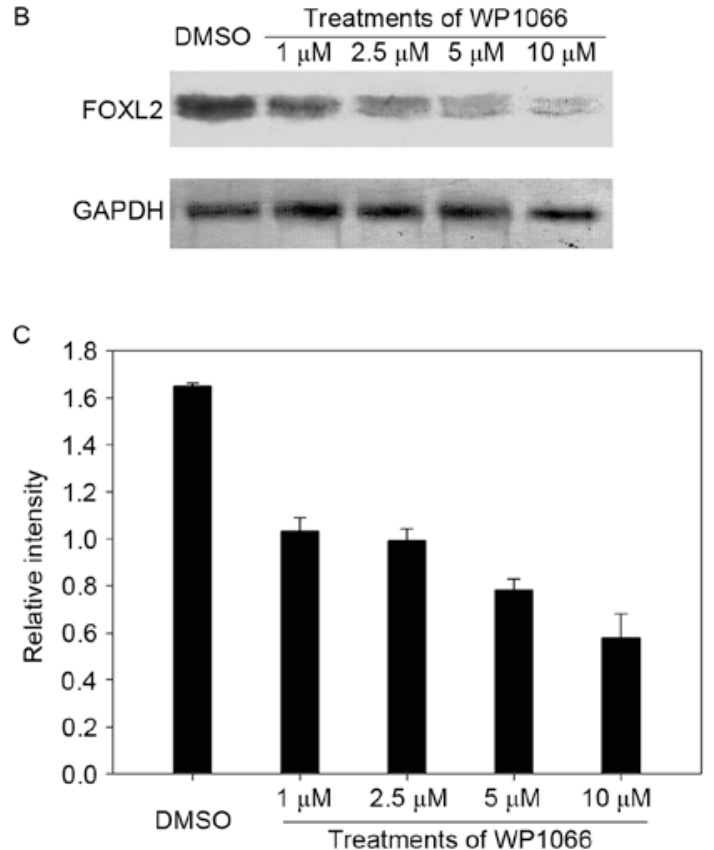

Figure 4. FOXL2 was downregulated by the STAT3 inhibitor, WP1066. (A) HeLa cells in 24-well plates were transfected with pGL3-Basic or pGL3-Enhancer vector plasmids containing the fusion promoter of FOXL2 (Basic-Pro and Enhancer-Pro). All HeLa cells were cotransfected with the Renilla luciferase reporter plasmid (pRL-TK) as a control for transfection efficiency using Lipofectamine ${ }^{\circledR} 3000$ reagent. A total of $24 \mathrm{~h}$ later, the STAT3 inhibitors C188-9 and WP1066 were applied at a final concentration $10 \mu \mathrm{M}$. Cells with no treatment served as the control group and those treated with DMSO served as the negative control. The luciferase activity was detected at $6 \mathrm{~h}$ and normalized by constitutive Renilla luciferase. Each column represents the mean \pm standard error of the mean of at least three replicates and the whole experiment was repeated 3 times. ${ }^{* * *} \mathrm{P}<0.005$, as indicated. (B) HeLa cells were treated with the indicated concentrations of WP1066 for $48 \mathrm{~h}$ and proteins were subjected to western blotting. Cells treated with DMSO served as the control and anti-GAPDH antibody was used as a loading control. (C) Blot were semi-quantified using densitometry and protein expression was normalized to GAPDH. Each column represents the mean \pm standard error of the mean of three replicates. FOXL2, forkhead box L; STAT3, signal transducer and activator of transcription 3; DMSO, dimethyl sulfoxide.

that although the concentration of DMSO was low $(<0.05 \%)$, it may suppress the viability of HeLa cells (Fig. 5). In a previous study, similar toxicity was observed in a retinal neuronal cell line (33). Thus, caution is required when DMSO is used as a control treatment in future studies using cell lines. 
A
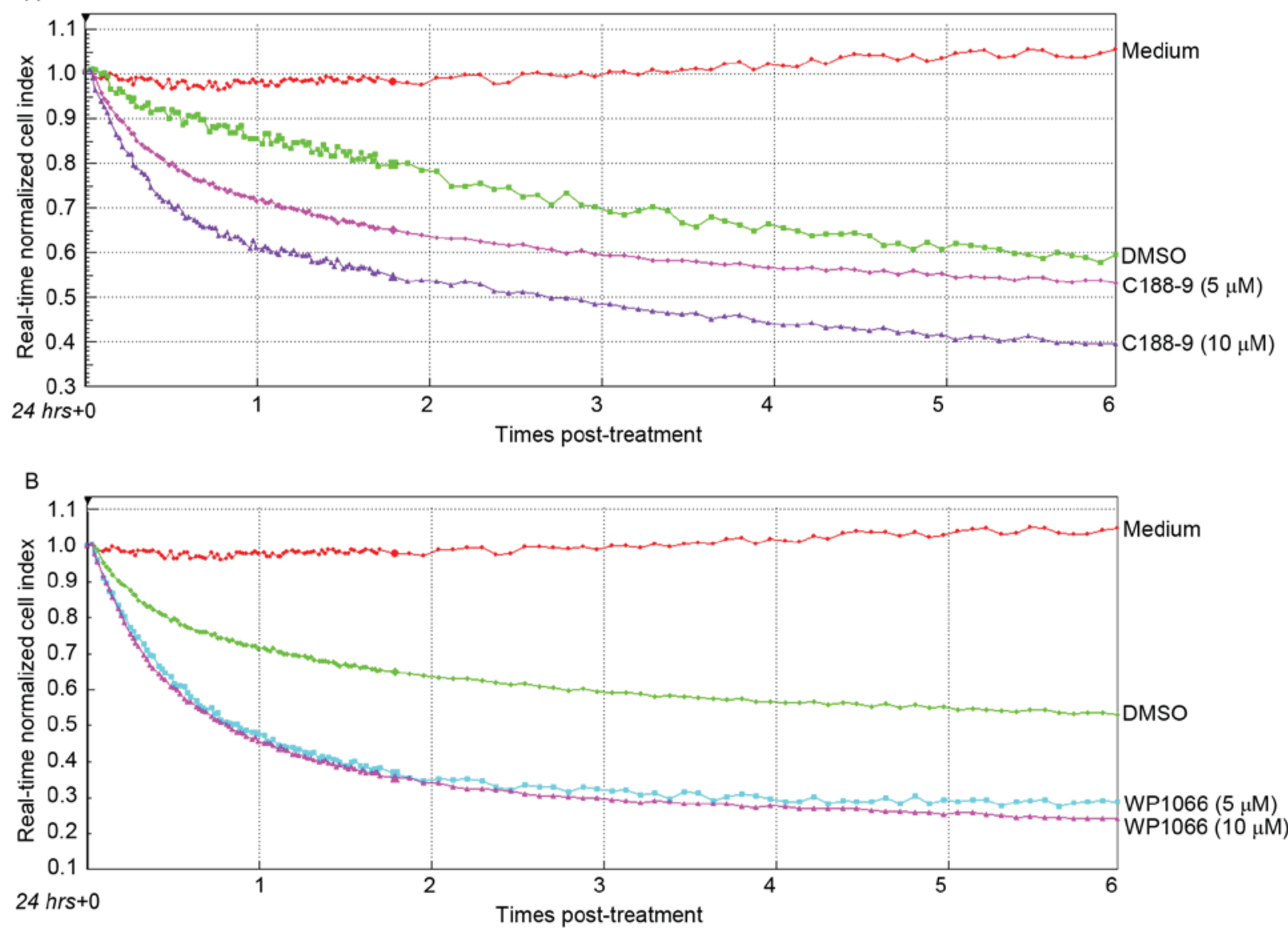

Figure 5. Real-time cell viability was detected following treatment with STAT3 inhibitor. Normal HeLa cells were plated onto an electrode-containing plate (e-plate) where they adhered for $24 \mathrm{~h}$, and then were treated with various STAT3 inhibitors (5 or 10 $\mu \mathrm{M})$; (A) C188-8 and (B) WP1066, while cells treated with DMSO served as the negative control. Cell viability was tracked using the xCelligence real-time viability system and each experiment was repeated at least two times. STAT3, signal transducer and activator of transcription 3; DMSO, dimethyl sulfoxide.

A previous study demonstrated that FOXL2 regulates certain genes, which are directly or indirectly involved in cell proliferation and apoptosis, such as tumor necrosis factor receptor 1 (TNF-Rl), FAS or TNF-related apoptosis-inducing ligand receptor (TRAIL-R) (34), which was similar to the results presented in Figs. 4 and 5, where cell proliferation was markedly inhibited subsequent to HeLa cells being exposed to STAT3 inhibitors. Similarly, Cheng et al (25) identified that FOXL2 activates the expression of the GNRH receptor in human and mouse GCs, which may exert a pro-apoptotic role.

In conclusion, the promoter of FOXL2 was successfully cloned and registered in Genbank (GenBank accession no. KR030055), and LUC activity was observed to be significantly induced by the promoter. Notably, the current results demonstrated for the first time that FOXL2 was regulated by STAT3, according to the DLR and western blot analysis. In addition, cell viability was revealed to be suppressed by DMSO even at a low concentration.

\section{Acknowledgements}

The authors would like to thank Dr Shaopeng Yuan for kindly providing the inhibitors of STAT3. The present study was supported by the National Natural Foundation of China (grant nos. 81501683 and 81471880), Natural Science Foundation of Shandong Province (grant no. ZR2015HL057) and the Doctoral Foundation of Weifang Medical University (Weifang, China).

\section{References}

1. Verdin $\mathrm{H}$ and De Baere E: FOXL2 impairment in human disease. Horm Res Paediatr 77: 2-11, 2012.

2. Rosario R, Cohen PA and Shelling AN: The role of FOXL2 in the pathogenesis of adult ovarian granulosa cell tumours. Gynecol Oncol 133: 382-387, 2014.

3. Georges A, Auguste A, Bessière L, Vanet A, Todeschini AL and Veitia RA: FOXL2: A central transcription factor of the ovary. J Mol Endocrinol 52: R17-R33, 2013.

4. Cocquet J, Pailhoux E, Jaubert F, Servel N, Xia X, Pannetier M, De Baere E, Messiaen L, Cotinot C, Fellous M and Veitia RA: Evolution and expression of FOXL2. J Med Genet 39: 916-921, 2002.

5. Uhlenhaut NH, Jakob S, Anlag K, Eisenberger T, Sekido R, Kress J, Treier AC, Klugmann C, Klasen C, Holter NI, et al: Somatic sex reprogramming of adult ovaries to testes by FOXL2 ablation. Cell 139: 1130-1142, 2009.

6. Garcia-Ortiz JE, Pelosi E, Omari S, Nedorezov T, Piao Y, Karmazin J, Uda M, Cao A, Cole SW, Forabosco A, et al: Fox12 functions in sex determination and histogenesis throughout mouse ovary development. BMC Dev Biol 9: 36, 2009.

7. Qian X, Shu A, Qin W, Xing Q, Gao J, Yang J, Feng G and He L: A novel insertion mutation in the FOXL2 gene is detected in a big Chinese family with blepharophimosis-ptosis-epicanthus inversus. Mutat Res 554: 19-22, 2004. 
8. Li WX, Wang XK, Sun Y, Wang YL, Lin LX and Tang SJ: A novel mutation in the FOXL2 gene in a Chinese family with blepharophimosis, ptosis, and epicanthus inversus syndrome. Zhonghua Yi Xue Yi Chuan Xue Za Zhi 22: 372-375, 2005

9. Yu HC, Geiger EA, Medne L, Zackai EH and Shaikh TH: An individual with blepharophimosis-ptosis-epicanthus inversus syndrome (BPES) and additional features expands the phenotype associated with mutations in KAT6B. Am J Med Genet A 164A: 950-957, 2014

10. Duffin K, Bayne RA, Childs AJ, Collins C and Anderson RA: The forkhead transcription factor FOXL2 is expressed in somatic cells of the human ovary prior to follicle formation. Mol Hum Reprod 15: 771-777, 2009.

11. Caburet S, Georges A, L'Hôte D, Todeschini AL, Benayoun BA and Veitia RA: The transcription factor FOXL2: At the crossroads of ovarian physiology and pathology. Mol Cell Endocrinol 356 : 55-64, 2012.

12. Auguste A, Chassot AA, Grégoire EP, Renault L, Pannetier M, Treier M, Pailhoux E and Chaboissier MC: Loss of R-spondin1 and Fox12 amplifies female-to-male sex reversal in XX mice. Sex Dev 5: 304-317, 2011

13. Dai A, Sun H, Fang T, Zhang Q, Wu S, Jiang Y, Ding L, Yan G and $\mathrm{Hu}$ Y: MicroRNA-133b stimulates ovarian estradiol synthesis by targeting Fox12. FEBS Lett 587: 2474-2482, 2013.

14. Luo Y, Wu X, Ling Z, Yuan L, Cheng Y, Chen J and Xiang C: microRNA133a targets Fox12 and promotes differentiation of C2C12 into myogenic progenitor cells. DNA Cell Biol 34: 29-36, 2015.

15. Eiring AM, Page BD, Kraft IL, Mason CC, Vellore NA, Resetca D, Zabriskie MS, Zhang TY, Khorashad JS, Engar AJ, et al: Combined STAT3 and BCR-ABL1 inhibition induces synthetic lethality in therapy-resistant chronic myeloid leukemia. Leukemia 29: 586-597, 2015.

16. Takeda K, Kaisho T, Yoshida N, Takeda J, Kishimoto T and Akira S: Correction: Stat3 activation is responsible for IL-6-dependent T cell proliferation through preventing apoptosis: Generation and characterization of T cell-specific Stat3-deficient mice. J Immunol 194: 3526, 2015.

17. Lee HJ, Zhuang G, Cao Y, Du P, Kim HJ and Settleman J: Drug resistance via feedback activation of Stat 3 in oncogene-addicted cancer cells. Cancer Cell 26: 207-221, 2014

18. Furqan M, Akinleye A, Mukhi N, Mittal V, Chen Y and Liu D: STAT inhibitors for cancer therapy. J Hematol Oncol 6: 90, 2013

19. Wang T, Li F and Tang S: MiR-30a upregulates BCL2A1, IER3 and cyclin D2 expression by targeting FOXL2. Oncol Lett 9 : 967-971, 2015

20. Pisarska MD, Barlow G and Kuo FT: Minireview: Roles of the forkhead transcription factor FOXL2 in granulosa cell biology and pathology. Endocrinology 152: 1199-1208, 2011

21. Kashimada K, Pelosi E, Chen H, Schlessinger D, Wilhelm D and Koopman P: FOXL2 and BMP2 act cooperatively to regulate follistatin gene expression during ovarian development. Endocrinology 152: 272-280, 2011.
22. Rosario R, Araki H, Print CG and Shelling AN: The transcriptional targets of mutant FOXL2 in granulosa cell tumours. PLoS One 7: e46270, 2012.

23. Rosario R, Blenkiron C and Shelling AN: Comparative study of microRNA regulation on FOXL2 between adult-type and juvenile-type granulosa cell tumours in vitro. Gynecol Oncol 129: 209-215, 2013.

24. Tran S, Lamba P, Wang Y and Bernard DJ: SMADs and FOXL2 synergistically regulate murine FSHbeta transcription via a conserved proximal promoter element. Mol Endocrinol 25: 1170-1183, 2011.

25. Cheng JC, Klausen C and Leung PC: Overexpression of wild-type but not $\mathrm{C} 134 \mathrm{~W}$ mutant FOXL2 enhances GnRH-induced cell apoptosis by increasing $\mathrm{GnRH}$ receptor expression in human granulosa cell tumors. PLoS One 8: e55099, 2013.

26. Benayoun BA, Batista F, Auer J, Dipietromaria A, L'Hôte D, De Baere E and Veitia RA: Positive and negative feedback regulates the transcription factor FOXL2 in response to cell stress: Evidence for a regulatory imbalance induced by disease-causing mutations. Hum Mol Genet 18: 632-644, 2009.

27. Staniszewska AD, Pensa S, Caffarel MM, Anderson LH, Poli V and Watson CJ: Stat 3 is required to maintain the full differentiation potential of mammary stem cells and the proliferative potential of mammary luminal progenitors. PLoS One 7: e52608, 2012.

28. Rokavec M, Öner MG, Li H, Jackstadt R, Jiang L, Lodygin D, Kaller M, Horst D, Ziegler PK, Schwitalla S, et al: IL-6R/STAT3/miR-34a feedback loop promotes EMT-mediated colorectal cancer invasion and metastasis. J Clin Invest 124 1853-1867, 2014

29. Iwamaru A, Szymanski S, Iwado E, Aoki H, Yokoyama T, Fokt I, Hess K, Conrad C, Madden T, Sawaya R, et al: A novel inhibitor of the STAT3 pathway induces apoptosis in malignant glioma cells both in vitro and in vivo. Oncogene 26: 2435-2444, 2007.

30. Daniel JM, Dutzmann J, Bielenberg W, Widmer-Teske R, Gündüz D, Hamm CW and Sedding DG: Inhibition of STAT3 signaling prevents vascular smooth muscle cell proliferation and neointima formation. Basic Res Cardiol 107: 261, 2012.

31. Redell MS, Ruiz MJ, Alonzo TA, Gerbing RB and Tweardy DJ: Stat3 signaling in acute myeloid leukemia: Ligand-dependent and -independent activation and induction of apoptosis by a novel small-molecule Stat3 inhibitor. Blood 117: 5701-5709, 2011.

32. Gad SE and Sullivan DW: Dimethyl Sulfoxide (DMSO). In: Encyclopedia Toxicol. Wexler P (ed). 3rd edition. Academic Press/Elsevier, London, pp166-168, 2014.

33. Galvao J, Davis B, Tilley M, Normando E, Duchen MR and Cordeiro MF: Unexpected low-dose toxicity of the universal solvent DMSO. FASEB J 28: 1317-1330, 2014

34. Kim JH, Yoon S, Park M, Park HO, Ko JJ, Lee K and Bae J: Differential apoptotic activities of wild-type FOXL2 and the adult-type granulosa cell tumor-associated mutant FOXL2 (C134W). Oncogene 30: 1653-1663, 2011. 